\title{
SEGURANÇA DO PACIENTE PEDIÁTRICO NO PROCESSO DE ADMINISTRAÇÃO DE MEDICAMENTO ENDOVENOSO
}

Cristina Oliveira da Costa ${ }^{1}$

Thais Lima Vieira de Souza ${ }^{1}$

Érica Oliveira Matias ${ }^{1}$

Sabrina de Souza Gurgel ${ }^{1}$

Rafaela de Oliveira Mota

Francisca Elisângela Teixeira Lima

\author{
https://orcid.org/0000-0002-2183-6224 \\ https://orcid.org/0000-0002-2161-2327 \\ https://orcid.org/0000-0002-6756-6223 \\ https://orcid.org/0000-0002-2180-5946 \\ https://orcid.org/0000-0003-1586-6678 \\ https://orcid.org/0000-0002-7543-6947
}

Objetivo: Avaliar o processo de preparo e administração de medicamentos endovenosos em um hospital pediátrico. Métodos: Estudo transversal de natureza quantitativa. Foram realizadas 135 observações do processo de preparo e administração de medicamentos por via endovenosa em crianças. Para a avaliação do desempenho dos profissionais na execução de cada ação do processo determinou-se o Índice de Positividade para Qualidade da Assistência. A análise foi através de estatística descritiva obedecendo aos preceitos éticos. Resultados: Na etapa anterior ao preparo dos medicamentos todas as ações obtiveram desempenho satisfatório ( $\geq 70 \%$ ). Das quinze ações do preparo dos medicamentos, nove foram satisfatórias ( $\geq 70 \%$ ). Durante e após a administração das seis das sete ações obtiveram desempenho satisfatório ( $\geq 70 \%)$. Conclusão: Os achados referentes ao estudo nos permitem evidenciar que existem falhas no processo de preparo e administração de medicamentos.

Descritores: Segurança do Paciente; Enfermagem Pediátrica; Pediatria.

\section{PEDIATRIC PATIENT SAFETY IN THE INTRAVENOUS DRUG ADMINISTRATION PROCESS}

Objective: To evaluate the process of preparation and administration of intravenous drugs in a pediatric hospital. Methods: Cross-sectional study of a quantitative nature. 135 observations were made of the process of preparation and administration of drugs intravenously in children. To assess the performance of professionals in the execution of each action in the process, the Positivity Index for Quality of Care was determined. The analysis was through descriptive statistics obeying the ethical precepts. Results: In the step prior to the preparation of the medications, all actions achieved satisfactory performance ( $\geq 70 \%)$. Of the fifteen actions for preparing medications, nine were satisfactory ( $\geq 70 \%)$. During and after the administration of six of the seven actions, they obtained satisfactory performance ( $\geq 70 \%)$. Conclusion: The findings regarding the study allow us to evidence that there are flaws in the process of preparing and administering medications.

Descriptors: Patient Safety; Pediatric Nursing; Pediatrics.

\section{SEGURIDAD DEL PACIENTE PEDIÁTRICO EN EL PROCESO DE ADMINISTRACIÓN DE DROGAS INTRAVENOSAS}

\section{RESUMEN}

Objetivo: evaluar el proceso de preparación y administración de drogas intravenosas en un hospital pediátrico. Métodos: estudio transversal de naturaleza cuantitativa. Se hicieron 135 observaciones sobre el proceso de preparación y administración de drogas por vía intravenosa en niños. Para evaluar el desempeño de los profesionales en la ejecución de cada acción en el proceso, se determinó el Índice de Positividad para la Calidad de la Atención. El análisis fue a través de estadisticas descriptivas que obedecen los preceptos éticos. Resultados: En el paso previo a la preparación de los medicamentos, todas las acciones lograron un desempeño satisfactorio ( $\geq 70 \%$ ). De las quince acciones para preparar medicamentos, nueve fueron satisfactorias ( $\geq 70 \%)$. Durante y después de la administración de seis de las siete acciones, obtuvieron un desempeño satisfactorio ( $\geq 70 \%)$. Conclusión: Los hallazgos con respecto al estudio nos permiten evidenciar que hay fallas en el proceso de preparación y administración de medicamentos.

Descriptores: Seguridad del paciente; Enfermería Pediátrica; Pediatría.

Universidade Federal do Ceará, CE, Brasil.

Autor Correspondente: Cristina Oliveira da Costa - Email: cristinaenfermagemufc@gmail.com

Recebido: 31/03/2020 - Aceito: 14/05/2020 


\section{INTRODUÇÃO}

A Segurança do Paciente no ambiente hospitalar tem sido tema de grande relevância nos últimos anos. Na atualidade, a busca pela melhoria da qualidade da assistência em saúde para promoção da segurança do paciente vem gerando melhoria dos resultados em diversos serviços de saúde mundialmente.

A Organização Mundial da Saúde (OMS), em 2004. apresentou por meio da Aliança Mundial para a Segurança do Paciente, diretrizes para a construção de um cuidado seguro e de qualidade para a população. Essa aliança tem o objetivo de despertar a consciência profissional e o comprometimento político para uma melhor segurança na assistência à saúde e indução de boas práticas assistenciais ${ }^{(1)}$.

Em 2008, no Brasil, a Rede Brasileira de Enfermagem e Segurança do Paciente (REBRAENSP) foi pioneira na discussão do tema e trouxe visibilidade para a enfermagem. O Ministério da Saúde (MS), no ano de 2013, apresenta o Programa Nacional de Segurança do Paciente através da Portaria № 529 que institui o tema como política de saúde no cenário brasileiro ${ }^{(2-4)}$

Corroborando com o Programa Nacional de Segurança do Paciente, o Ministério da Saúde em 2013 aprovou e instituiu seis Protocolos Básicos para a Segurança do Paciente, sendo utilizado neste estudo o Protocolo de prescrição, uso e administração de medicamentos. Assim, há avanços na construção da cultura da segurança do paciente na atenção à saúde da criança hospitalizada, com estabelecimento de estratégias para promoção do cuidado seguro na enfermagem pediátrica ${ }^{(3-4)}$.

A instituição de programas de educação permanente tem se mostrado efetiva na prevenção de erros de medicação. Em um estudo que discutiu dados referentes à epidemiologia dos erros de medicação na pediatria mostram que um dos principais fatores que contribuem para que estes ocorram é a falta de acesso a informações sobre os medicamentos por parte de todos os profissionais envolvidos no processo. $\mathrm{O}$ uso de protocolos assistenciais no planejamento da assistência permite a implementação mais segura da terapia medicamentosa ${ }^{(5)}$.

Dentro da complexidade do cuidado, erros relacionados à medicação apontam para a falta de políticas de saúde e da indústria farmacêutica voltadas ao atendimento das especificidades da população pediátrica(6).

Um estudo realizado no setor de pediatria de um hospital em São Paulo verificou que os eventos adversos intra-hospitalares graves relacionados a erros de medicação estão relacionados a falhas técnicas, humanas e a causas organizacionais ${ }^{(7)}$

Um dos primeiros estudos prospectivos sobre erros de medicação em crianças foi publicado em 1987, no qual farmacêuticos revisaram prescrições de medicamentos de dois hospitais pediátricos, sendo detectada taxa de 0,49 erros por 100 medicamentos prescritos ${ }^{(4)}$

Um estudo realizado em um hospital universitário no sul do Brasil ratifica que dos 115 eventos notificados no período do estudo, 23 foram relacionados ao processo medicamentoso. Nesse sentido, é imprescindível a análise dos fatores causais dos incidentes, de modo que possam ser identificadas medidas para a prevenção de danos ao paciente ${ }^{(6)}$.

Estudo realizado em um hospital pediátrico no ano de 2017 sobre administração de medicamentos, demonstra que de 18 ações de observações do processo de preparo e administração de medicamentos em crianças hospitalizadas apenas 8 tiveram resultados satisfatório, maior que $70 \%$ de frequência ${ }^{(8)}$.

Com base nessa contextualização, as questões de pesquisa foram: como é realizado o processo de preparo e administração de medicamentos endovenosos em um hospital pediátrico?

Assim, teve-se como objetivo avaliar o processo de preparo e administração de medicamentos endovenosos em um hospital pediátrico.

\section{MÉTODOS}

\section{Tipo de estudo}

Estudo observacional, transversal, de abordagem quantitativa, desenvolvido em um hospital pediátrico de grande porte, da atenção terciária, da rede estadual em Fortaleza, Ceará. A instituição dispõe de equipe multiprofissional de profissionais de saúde e dentre estes profissionais de enfermagem: enfermeiros, técnicos de enfermagem e auxiliares de enfermagem. Os setores selecionados para a realização do estudo foram as unidades abertas de internação que englobam as seguintes especialidades: neurologia, cardiologia, pneumologia, gastroenterologia, nefrologia, pediatria geral e cirúrgica; sendo a escolha do cenário do estudo uma solicitação da própria instituição. A instituição conta com setor de educação permanente e com protocolos para preparo e administração de medicamentos, sendo realizadas capacitações periódicas para os profissionais sobre o tema.

\section{População}

Foram realizadas 135 observações sistemáticas de profissionais de enfermagem realizando o processo de prepa- 
ro e administração de medicamentos por via endovenosa em crianças de $\mathrm{O}$ a 10 anos atendidas na referida instituição. Participaram do estudo 11 técnicos de Enfermagem.

\section{Critérios de seleção}

Os profissionais foram selecionados a partir dos seguintes critérios de inclusão: ser profissional de Enfermagem, atuar na administração de medicamentos pela via endovenosa e estar de serviço no período da coleta de dados. Já como critérios de exclusão, temos: estar de férias, licença ou afastado nos dias que serão realizadas as observações; e recusar participar da pesquisa.

\section{Coleta de dados}

A coleta dos dados foi realizada nos meses de junho a outubro de 2017, nos turnos manhã, tarde e noite, englobando todos os dias da semana. Por meio da observação não participante, direta e sistemática das ações antes, durante e após a administração do medicamento por meio de um instrumento do tipo check-list construido com base nas recomendações do Protocolo de Segurança na prescrição, uso e administração de medicamentos $^{(2)}$. A coleta dos dados foi realizada pela pesquisadora e por bolsistas de iniciação científica treinados para a coleta dos dados nas unidades abertas de internação da instituição.

Para tanto, foi realizada uma entrevista e observações diretas e não participantes das ações desenvolvidas pelos técnicos de Enfermagem durante o processo de preparo e administração de medicamentos pela via endovenosa na pediatria.

No que concerne à observação da etapa de higienização das mãos, foi analisada a execução da técnica correta, seguindo o passo-a-passo preconizado pela Agência Nacional de Vigilância Sanitária - ANVISA ${ }^{(9)}$.

\section{Análise e tratamento dos dados}

Os dados coletados no estudo foram armazenados em banco de dados produzido pelos pesquisadores no Microsoft Excel do Windows 2010, processados e analisados de forma descritiva e de acordo com as recomendações do Protocolo de Segurança na Prescrição, Uso e Administração de medicamentos ${ }^{(2)}$.

Para a avaliação do desempenho dos profissionais na execução de cada ação do processo, determinou-se o Índice de Positividade (IP) para Qualidade da Assistência (OA), que determina desejável (100\% IP), adequado (90 a 99\% IP), seguro (80 a $89 \%$ IP), limitrofe (71 a $79 \%$ IP) e sofrivel ( $\leq$ $70 \%$ IP)(10).
No Quadro 1 estão dispostas as ações observadas antes, durante e após o processo de preparo e administração dos medicamentos.

Quadro 1 - Distribuição das ações observadas antes, durante e após o processo de preparo e administração de medicamentos, Brasil, 2017.

Ações observadas antes do preparo dos medicamentos

- Lê a prescrição médica

- Consegue entender a grafia da prescrição médica

- Confere o nome do paciente relacionado com a prescrição

Ações observadas durante o preparo dos medicamentos

- Realiza limpeza e organiza a bancada

- Dispõe de bandeja ou cuba rim

- Usa equipamento de proteção individual

- Escolhe seringa compativel com o volume a ser administrado

- Seleciona adequadamente a agulha para aspiração

- Confere o rótulo da medicação com a prescrição

- Verifica a integridade dos invólucros

- Faz a inspeção do frasco

- Observa data de validade do medicamento

- Faz desinfecção da ampola ou frasco-ampola

- Medicação reconstituída em água destilada ou soro fisiológico

- Medicação preparada para administração em bolus

- Medicação preparada para infusão contínua

- Identifica a medicação preparada

- Descarta adequadamente os materiais utilizados

Ações observadas após o preparo e durante a administração dos medicamentos

- Confere medicamento relacionado com a prescrição

- Confere o nome do paciente relacionado com a prescrição

- Instala medicação conforme prescrição médica

- Mantém paciente confortável

- Registra/checa imediatamente no prontuário/prescrição a adminis tração do medicamento

- Monitora paciente

- Orienta paciente/acompanhante 


\section{Aspectos éticos}

O estudo foi aprovado pelo Comitê de Ética em Pesquisa da Universidade Federal do Ceará, sob parecer 2.043.123/2017, seguindo os preceitos éticos e respeitando as normas da Resolução do Conselho Nacional de Saúde (CNS) no 466/2012 e seus complementos ${ }^{(11)}$.

\section{RESULTADOS}

A distribuição acerca do processo de preparo e administração de medicamentos endovenosos está exposta em ações antes, durante e após o procedimento. Conforme tabelas 1, 2 e 3.

Tabela 1 - Distribuição das ações referentes as ações executadas antes do preparo dos medicamentos, Brasil, 2017.

\begin{tabular}{|l|l|l|l|}
\hline Ação observada & N=135 & $\%$ & IP \\
\hline Lê a prescrição médica & 132 & 97,8 & Adequado \\
$\begin{array}{l}\text { Consegue entender a grafia da } \\
\text { prescrição médica }\end{array}$ & 135 & 100 & Desejável \\
$\begin{array}{l}\text { Confere o nome do paciente } \\
\text { relacionado com a prescrição }\end{array}$ & 110 & 81,4 & Seguro \\
\hline
\end{tabular}

A ação "lê a prescrição médica" foi realizada pela maioria dos técnicos de Enfermagem (97,8\%), caracterizando desempenho adequado. A ação "consegue entender a grafia da prescrição médica" foi alcançada em todas as leituras observadas, obtendo desempenho desejável. O fato de a instituição disponibilizar um sistema informatizado para a realização das prescrições eletrônicas foi fator positivo para o bom desempenho dos profissionais nesta etapa.

Para a ação "confere nome da criança relacionando com a prescrição" em 18,6\% das observações constatou-se que o profissional de Enfermagem não checou se era o paciente certo, tendo essa ação desempenho seguro.

Destaca-se que das 135 observações, em 99,3\% foi realizado o descarte adequado dos materiais utilizados durante o preparo, corroborando com o gerenciamento de residuos de saúde adequadamente.

A tabela 2 expõe as ações referentes ao preparo das medicações.

Tabela 2 - Distribuição das ações referentes as ações executadas durante o preparo dos medicamentos, Brasil, 2017

\begin{tabular}{l|l|l|l|}
\hline Ação observada & $\mathrm{N}=135$ & $\%$ & IP \\
\hline $\begin{array}{l}\text { Realiza limpeza e organiza a } \\
\text { bancada }\end{array}$ & $119 \quad 88,1 \quad$ Seguro
\end{tabular}

\begin{tabular}{|c|c|c|c|}
\hline Dispõe de bandeja ou cuba rim & 134 & 99,3 & Adequado \\
\hline $\begin{array}{l}\text { Usa equipamento de proteção } \\
\text { individual }\end{array}$ & 132 & 97,7 & Adequado \\
\hline $\begin{array}{l}\text { Escolhe seringa compativel } \\
\text { com o volume a ser administra- } \\
\text { do }\end{array}$ & 134 & 99,3 & Adequado \\
\hline $\begin{array}{l}\text { Seleciona adequadamente a } \\
\text { agulha para aspiração }\end{array}$ & 121 & 89,6 & Seguro \\
\hline $\begin{array}{l}\text { Confere o rótulo da medicação } \\
\text { com a prescrição }\end{array}$ & 115 & 85,2 & Seguro \\
\hline $\begin{array}{l}\text { Verifica a integridade dos } \\
\text { invólucros }\end{array}$ & 89 & 65,9 & Sofrivel \\
\hline Faz a inspeção do frasco & 80 & 59,3 & Sofrivel \\
\hline $\begin{array}{l}\text { Observa data de validade do } \\
\text { medicamento }\end{array}$ & 12 & 8,9 & Sofrivel \\
\hline $\begin{array}{l}\text { Faz desinfecção da ampola ou } \\
\text { frasco-ampola }\end{array}$ & 28 & 20,7 & Sofrivel \\
\hline $\begin{array}{l}\text { Medicação reconstituida em } \\
\text { água destilada ou soro fisio- } \\
\text { lógico }\end{array}$ & 132 & 97,8 & Adequado \\
\hline $\begin{array}{l}\text { Medicação preparada para } \\
\text { administração em bolus }\end{array}$ & 54 & 40 & Sofrivel \\
\hline $\begin{array}{l}\text { Medicação preparada para } \\
\text { infusão continua }\end{array}$ & 81 & 60 & Sofrivel \\
\hline $\begin{array}{l}\text { Identifica a medicação pre- } \\
\text { parada }\end{array}$ & 116 & 85,9 & Seguro \\
\hline $\begin{array}{l}\text { Descarta adequadamente os } \\
\text { materiais utilizados }\end{array}$ & 134 & 99,3 & Adequado \\
\hline
\end{tabular}

Na maioria das observações da ação “realiza limpeza e organização da bancada", os profissionais de Enfermagem executaram a limpeza da bancada e organizaram o material para o preparo do medicamento, representando $88,1 \%$ do total de observações.

Em 99,3\% das observações, identificou-se que o profissional dispunha de bandeja ou cuba rim para utilizar no processo de preparo e administração dos medicamentos endovenosos.

A ação "usa equipamento de proteção individual (gorro, máscara e luva)", no total de $97,7 \%$ foi evidenciado que $28,2 \%$ usavam adequadamente os equipamentos de proteção individual, 69,6\% usavam de forma parcial.

Os itens "escolhe seringa compativel com o volume a ser administrado" e "seleciona adequadamente agulhas para aspiração", apresentaram percentual 99,3\% e 89,6\%, representando adequado e seguro, respectivamente. 
Ao examinar o frasco ou a ampola utilizado, devem-se observar as informações presentes neles e realizar sua desinfecção antes da realização do preparo da medicação, conforme exposto na Tabela 2.

A ação "confere o rótulo da medicação com a prescrição" obteve $85,2 \%$ de execução durante os preparos observados, sendo a única ação referente à ampola ou frasco-ampola realizada de maneira adequada. As outras ações referentes ao frasco de medicação atingiram índices sofriveis de execução: em 65,9\% foi verificada a integridade dos invólucros; em 59,3\% foi realizada a inspeção do frasco para observar possiveis partículas, alteração da cor, rachaduras e/ou vazamentos; em $8,9 \%$ observou-se a data de validade do medicamento; e em $20,7 \%$ foi feita a desinfecção da ampola ou frasco-ampola.

O resultado foi adequado para as observações das ações: "medicação reconstituída em água destilada ou soro fisiológico, respeitando os princípios de prevenção da infecção hospitalar", sendo realizada em $97,8 \%$ do total. A utilização de frascos de água destilada estéril com $5 \mathrm{ml}$ de volume, diminui os riscos de contaminação quando comparado com frascos compartilhados para várias diluições, contribuindo para desempenho adequado.

Em relação à ação "identifica a medicação preparada", em $85,9 \%$ das observações foi constatada tal ação, esta ação está diretamente relacionada com a segurança do paciente visando evitar a possibilidade de erros durante a administração de medicamentos.

Tabela 3 - Distribuição das ações referentes a administração dos medicamentos pela via endovenosa, Brasil, 2017.

\begin{tabular}{l|l|l|l|}
\hline Ação observada & N=135 & $\%$ & IP \\
\hline $\begin{array}{l}\text { Confere medicamento relacionado } \\
\text { com a prescrição }\end{array}$ & 125 & 92,6 & Seguro \\
$\begin{array}{l}\text { Confere o nome do paciente relacio- } \\
\text { nado com a prescrição }\end{array}$ & 108 & 80 & Adequado \\
$\begin{array}{l}\text { Instala medicação conforme prescri- } \\
\text { ção médica }\end{array}$ & 135 & 100 & Desejável \\
$\begin{array}{l}\text { Mantém paciente confortável } \\
\text { Registra/checa imediatamente no } \\
\text { prontuário/prescrição a administração } \\
\text { do medicamento }\end{array}$ & 113 & 87,4 & Adequado \\
\hline $\begin{array}{l}\text { Monitora paciente } \\
\text { Orienta paciente/acompanhante }\end{array}$ & 78 & 57,8 & Sofrivel \\
\hline
\end{tabular}

A técnica de administração do medicamento pela via endovenosa mostrou que em 92,6\% das observações os profissionais de enfermagem após o preparo das soluções conferiram o medicamento com as informações presentes na prescrição médica, e em apenas $80 \%$ o profissional conferiu o paciente com o nome que consta na prescrição.

Em todas as observações (100\%) foram instaladas as medicações conforme descrito na prescrição médica, mas em apenas $87,4 \%$ o profissional manteve o paciente de modo confortável.

As ações de registrar/checar imediatamente no prontuário/prescrição a administração do medicamento (83,7\%), de monitorar o paciente $(93,4 \%)$ e de registrar queixas e/ou reações adversas apresentadas pelo paciente (89,5\%) foram realizadas de forma seguras, adequada e segura, respectivamente; enquanto que a ação de orientar o paciente/ acompanhante $(57,8 \%)$ foi executada de maneira sofrível.

\section{DISCUSSÃO}

A Enfermagem tem como uma de suas responsabilidades e deveres inerentes à profissão, assegurar à pessoa, família e coletividade assistência livre de danos decorrentes de imperícia, negligência ou imprudência ${ }^{(12)}$

Uma das atividades mais desempenhadas pela equipe de enfermagem é a administração de medicamentos, sendo portanto necessária uma avaliação contínua desse processo para que os pacientes não venham a sofrer danos preveníveis decorrentes desse processo ${ }^{(13)}$

O papel do enfermeiro relativo à terapêutica medicamentosa tem sido foco de questionamentos. Isso ocorre devido ao conhecimento técnico científico relacionado a esta prática e ao distanciamento do enfermeiro, no que tange a supervisão do preparo e a administração de medicamentos, em virtude desta responsabilidade muitas vezes ser atribuida ao técnico ou auxiliar de enfermagem ${ }^{(14)}$.

Em relação à prescrição dos medicamentos é de suma importância que esta seja legivel, de entendimento universal para que todos os profissionais possam implementar a assistência prescrita de forma segura e de qualidade. No presente estudo as ações de ler a prescrição médica e entender a grafia, ou seja, o que está prescrito foi desempenhado de forma satisfatória. A prescrição médica é o documento de referência que norteia e influencia as demais etapas do processo de medicação. É instrumento essencial de comunicação entre os profissionais de saúde e tem importante papel na prevenção e ocorrência de erros ${ }^{(14)}$.

Em 2015, estudo realizado em um hospital pediátrico de Fortaleza no Ceará demonstra que os profissionais conseguiram ler a prescrição médica de forma a entender 
a grafia, tendo entendimento satisfatório da mesma. Em contrapartida, a ação de conferir o nome do paciente com a prescrição foi desempenhada de forma insatisfatória pela equipe de enfermagem com apenas 40,7\% das ações observadas ${ }^{(15)}$.

Estudo realizado em um hospital pediátrico no Ceará apontou que das 327 observações, apenas em 0,3\% os profissionais se apresentaram para as crianças e/ou responsáveis antes do procedimento, em 10,3\% realizaram a explicação do procedimento para a criança e/ou responsável, e em $18,3 \%$ a criança foi acalmada, o que pode contribuir para a realização inadequada do procedimento, visto que ao esclarecer o que será realizado, o paciente, principalmente o pediátrico, torna-se mais colaborativo e menos ansioso, temeroso e inseguro(8).

A ocorrência de erros na assistência em saúde podem ser relacionadas a falhas humanas, falhas do sistema ou até mesmo dos processos no desempenho dos cuidados diretos e indiretos em saúde, além disso o comportamento humano também influencia diretamente na segurança do paciente $\mathrm{p}^{(16)}$

Estudo retrospectivo que investigou um hospital filantrópico de ensino desenhou os processos no preparo e administração de medicação, sendo identificadas tecnologias preventivas no processo de medicação, algumas dessas de baixo custo como capacitação da equipe, identificação do leito do paciente (placa), identificação do paciente (pulseira), prescrição médica eletrônica, dupla checagem de medicamentos potencialmente perigosos, dentre outras. Sendo que a redução da incidência de erro de medicação nesta instituição foi proporcional aos recursos materiais, tecnológicos e custos implementa$\operatorname{dos}^{(17)}$.

A ação "realiza limpeza e organização da bancada" foi desempenhada em $88,1 \%$ das observações, sendo essa atitude um fator imprescindivel para facilitar o processo de trabalho e evitar possíveis equívocos durante a realização de tarefas.

Quando o ambiente é organizado, isso contribui para prevenir quaisquer eventos adversos relacionados à assistência em saúde, e quando afetados por fatores dificultadores, como escassez ou má utilização de materiais, disposição não funcional de mobiliários e multiplicidade de tarefas no espaço, inviabilizam a correta estruturação do sistema de medicação ${ }^{(18)}$.

Um estudo realizado em um hospital de ensino no Ceará observou a inadequação da organização do ambiente em $70,6 \%$ de suas observações. Além disso, realizaram testes para verificar a associação da organização no preparo com a escolha errada do medicamento nesta ação, demonstrando que existe associação entre as duas variáveis ( $p$-valor $=0,027)^{(16)}$

A ação "dispõe de bandeja ou cuba rim" esteve presente em 99,3\% das observações. A não utilização desses insumos para o transporte dos medicamentos preparados pode interferir na integridade físico-química e microbiológica, além de ser um fator potencial de risco biológico, pois o transporte manual representa risco de acidentes e de contaminação da solução(19,15)

Estudo da análise fotográfica de imagens relacionadas ao preparo de medicamentos realizados por profissionais de enfermagem de um hospital de ensino no Rio Grande do Norte verificou como resposta que os profissionais identificaram como inseguro no processo de preparo de medicamentos a sobre a fotografia que registra os medicamentos que serão administrados em horários próximos ou posteriores separados com copos de plástico apenas com etiquetas para identificação ou diluídos e acondicionados em seringas apenas com etiquetas de identificação, facilitando a troca de medicação e/ou administração no paciente de forma equivocada(20)

A utilização de equipamentos de proteção individual (EPI), considerados no estudo o gorro, a máscara e a luva de procedimento, foram observados o uso de um ou mais equipamentos de proteção individual em $97,8 \%$, sendo que em apenas $28,7 \%$ foram utilizados concomitante o gorro, a máscara e a luva de procedimento, nas demais observações os profissionais utilizaram apenas um EPI como o gorro ou dois equipamentos de proteção como o gorro e a máscara.

Para uma assistência de qualidade, faz-se necessário que processos de trabalho sejam revistos e que profissionais sejam capacitados e treinados. É importante também, que as instituições disponibilizem tecnologias que possam ajudar nesse aprimoramento ${ }^{(3)}$.

Diante desses achados, é imprescindivel que ações visando a segurança do paciente no processo de preparo e administração de medicamentos na pediatria pelos profissionais de Enfermagem sejam implementadas de forma efetiva, por meio de educação permanente e capacitações periódicas sobre o preparo e administração seguros de medicamentos, com o intuito de minimizar a ocorrência de erros de medicação em todas as etapas desse processo, desde a leitura da prescrição até após a administração dos medicamentos com o monitoramento do paciente. 


\section{Limitações do estudo}

Este estudo possui limitações, uma vez que foi realizado em um único hospital, na cidade de Fortaleza e uma amostra de apenas 135 observações do procedimento de preparo e administração de medicamentos, o que impossibilita a generalização dos dados deste estudo, sendo necessária a realização de outros estudos pertinentes ao assunto.

\section{Contribuições para a prática}

Por meio deste estudo foi possível verificar do desempenho dos profissionais no processo de preparo e administração de medicamentos, com base nesse estudo, treinamentos da equipe de enfermagem podem ser planejados e implementados com base no que foi verificado nos resultados deste estudo, sendo necessária a realização de estudos posteriores para verificar o desempenho desses profissionais após treinamento.

\section{CONCLUSÃO}

O presente estudo permitiu analisar o processo de administração de medicamentos por via endovenosa por parte da equipe de enfermagem na pediatria. Os achados referentes ao estudo nos permite evidenciar que existem falhas no processo de preparo e administração de medicamentos, principalmente no que diz respeito ao momento de preparo dos medicamentos.
Constatou-se que das quinze ações avaliadas durante o preparo dos medicamentos, seis foram desempenhadas de forma sofrivel (IP igual ou menor que 70\%) segundo a classificação do índice de segurança.

Em suma, dentre as 35 ações que compõem o processo de administração de medicamentos pela via endovenosa, apenas três ações "consegue entender a grafia da prescrição médica" e "Instala medicação conforme prescrição médica" e "Monitora paciente" foram desempenhadas de forma desejável (IP = 100\%), enquanto que nove ações foram enquadradas como adequadas (IP entre 90 e 99\%), seis ações seguras (IP entre 80 e $89 \%$ ), e dezessete ações consideradas sofríveis (IP igual ou menor que 70\%). As ações desempenhadas de forma sofrivel (IP igual ou menor que 70\%) impactam diretamente na segurança do processo de preparo e administração dos medicamentos na pediatria.

\section{Contribuições dos autores:}

Concepção e/ou desenho do estudo: Souza TLV, Costa OC, Matias EO, Gurgel SS, Lima FET, Mota RO. Coleta, análise e interpretação de dados: Souza TLV, Costa OC, Matias EO, Gurgel SS, Lima FET, Mota RO. Redação e/ou crítica do manuscrito: Souza TLV, Costa OC, Matias EO, Gurgel SS, Lima FET, Mota RO. Aprovação da versão final a ser publicada: Souza TLV, Costa OC, Matias EO, Gurgel SS, Lima FET, Mota RO

\section{REFERÊNCIAS}

1. World Health Organization. World Alliance for Patient Safety: Forward Programme 2008-2009 [Internet]. Geneva: WHO: 2009 [cited 2019 Jul 29] Avaliable from: https:// apps.who.int/iris/handle/10665/70460

2. Ministério da Saúde (BR). Protocolo de Segurança na Prescrição, Uso e Administração de Medicamentos [Internet]. Brasilia; 2013 [citado 2019 Jul 25]. Disponivel em: https://www20.anvisa.gov.br/segurancadopaciente/index. php/publicacoes/item/seguranca-na-prescricao-uso-e-administracao-de-medicamentos

3. Wegner W, Silva MUM, Peres MA, Bandeira LE, Frantz $\mathrm{E}$, Botene CM, et al. Segurança do paciente no cuidado à criança hospitalizada: evidências para enfermagem pediátrica. Rev Gaúc Enferm. 2017; 28: e68020. Disponivel em: https://www.scielo.br/scielo.php?script=sci_abstract\&pi$\mathrm{d}=$ S198314472017000100504\&lng=en\&nrm=iso\&tlng=pt. DOI: http://dx.doi.org/10.1590/1983-1447.2017.01.68020
4. Ministério da Saúde (BR). Portaria n. 529, de 1 으 de abril de 2013. Institui o Programa Nacional de Segurança do Paciente (PNSP) [Internet]. Brasilia; 2013 [Citado 2019 Jun 30]. Disponivel em: http://www.saude.mt.gov.br/ upload/controle-infeccoes/pasta2/portaria-msgm-n-529-de-01-04-2013.pdf

5. Belela ASC, Pedreira MLG, Peterlini MAS. Erros de medicação em Pediatria. Rev Bras de Enferm. 2011; 64. Disponivel em: DOI: http://dx.doi.org/10.1590/S003471672011000300022

6. Bica TFS, Wegner W, Gerhardt LM, Predebom CM, Pedro ENR, Breigeiron MK. Characteristics of patient safety incidents notified in a pediatric intensive care unit. Rev Enferm Ufpe On Line. 2017; 11 Supl 10: S4206-16. Disponivel em: https://periodicos.ufpe.br/revistas/revistaenfermagem/ article/view/231184. DOI: http://dx.doi.org//0.5205/reuol. 10712-95194-3-SM.1110sup201726 
7. Lima MEA, Ribeiro EAF. Eventos adversos com medicamentos na pediatria: o papel da organização hospitalar. Cad Bras Ter Ocup. 2017; 24 (4): 927-933. Disponivel em: http://www.cadernosdeterapiaocupacional.ufscar.br/index.php/cadernos/article/view/1609/926. DOI: https:// dx.doi.org/10.4322/2526-8910.ctoRE0847

8. Souza TLV, Mota RO, Brito EAW, Farias LMVC, Matias EO, Lima FET. Segurança do paciente na administração de medicamento intramuscular em pediatria: avaliação da prática de enfermagem. Rev Gaúcha Enferm, 2017; 01: e2017-0002. Disponivel em: https://www.scielo.br/scielo. php?pid=S1983-14472018000100404Escript=sci_arttext. DOI: https://doi.org/10.1590/1983- 1447.2018.2017-0002

9. Ministério da Saúde (BR). Segurança do Paciente: higienização das mãos. [Internet]. Brasilia; 2013 [Citado 2019 Jun 30]. Disponivel em: https://www20.anvisa.gov.br/segurancadopaciente/index.php/publicacoes/item/seguranca-do-paciente-higienizacao-das-maos

10. Murassaki ACY, Versa GLGS. Bellucci Júnior JA. et al. Avaliação de cuidados na terapia intravenosa: desafio para a qualidade na enfermagem. Esc Anna Nery. 2013 17 (1): 11-6. Disponivel em: https://www.scielo.br/scielo. php?script=sci_arttext\&pid=S1414-81452013000100002. Acesso em: 02 Mai 2020

11. Ministério da Saúde (BR) Conselho Nacional de Saúde. Resolução n. 466, de 12 de dezembro de 2012. Dispõe sobre as diretrizes e normas regulamentadoras de pesquisas envolvendo seres humanos [Internet]. Brasilia; 2012 [Citado 2019 Jun 25]. Disponivel em: https://conselho. saude.gov.br/resolucoes/2012/Reso466.pdf

12. Souza MJ, Real DSS, Cunha ICKO, Bohomol E. Práticas seguras para administração de medicamentos: construção e validação de instrumento. Enferm Foco. 2017; 8 (4): 20-25. Disponivel em: http://revista.cofen.gov.br/index. php/enfermagem/article/view/973. Acesso em: 03 Mai 2020 .

13. Conselho Federal de Enfermagem. Resolução n. 564 , de 6 de dezembro de 2017. Dispõe sobre o Código de Ética dos Profissionais de Enfermagem [Internet]. Brasilia; 2017 [Citado 2019 Jun 25]. Disponivel em: http://www.cofen. gov.br/resolucao-cofen-no-5642017_59145.html
14. Tomasi YT, Souza GN, Bitencourt JVOV, Parker AG, Martini JG, Mancia JR. Atuação do enfermeiro na administração de medicamentos em uma instituição hospitalar: estudo descritivo. Enferm Foco. 2015; 1 (6): 06-11 Disponivel em: http://revista.cofen.gov.br/index.php/ enfermagem/article/view/569/251. Acesso em: 02 Mai 2020.

15. Pires AOM, Ferreira MBG, Nascimento KG, Felix MMS, Pires PS, Barbosa MH. Elaboration and Validation of the Medication Prescription Safety Checklist. Rev Lat Amer Enferm, 2017; 25: e2921. Disponivel em: https://www.scielo.br/scielo.php?script=sci_arttextEpid=S0104-11692017000100365. DOI: https://doi. org/10.1590/1518-8345.1817.2921

16. Matias, EO. Avaliação da prática de enfermagem no processo de administração de medicamento intravenoso na pediatria [Dissertação]. Fortaleza: Universidade Federal do Ceará; 2015. Disponivel em http://www.repositorio. ufc.br/bitstream/riufc/10530/1/2015_dis_eomatias.pdf Acesso em: 02 Mai 2020.

17. Vilela RPB, Jericó MC. Implantação de tecnologias para a prevenção de erros de medicação em hospital de alta complexidade: análise de custos e resultados. Einstein. 2019; 17 (4): 1-7. Disponivel em: https://www. scielo.br/pdf/eins/v17n4/pt_2317-6385-eins-17-04eGS4621.pdf. DOI: https://doi.org/10.31744/einstein_ journal/2019gs4621

18. Malagutti W, Roegrs H. Terapia Intravenosa: Atualidades. São Paulo: Martinari; 2012.

19. Pereira FGF, Aquino GA, Melo GAA, Praxedes CO, Caetano JA. Conformidades e não conformidades no preparo e administração de antibacterianos. Cog Enferm. 2016; 26( n.esp ): 188-198. Disponivel em: https:// revistas.ufpr.br/cogitare/article/view/45506. Acesso em: 02 Mai 2020.

20. Pereira CDFD, Tourino FSV, Santos VEP. Segurança do paciente: avaliação do sistema de medicação por enfermeiros utilizando análise fotográfica. Enferm Foco. 2016; 7 (1): 76-80. Disponivel em: http://revista.cofen.gov.br/index.php/enfermagem/article/view/672/290. Acesso em: 03 Mai 2020. 